\title{
Performance of penicillinase detection tests in Staphylococcus epidermidis: comparison of different phenotypic methods
}

Benjamin Aubry ${ }^{1}$, Carole Lemarié ${ }^{1}$, Rachel Chenouard ${ }^{1}$, Marie Kempf ${ }^{1,2}$, Matthieu Eveillard ${ }^{1,2}$ and Hélène Pailhoriès ${ }^{1,3^{*}}$ (iD

\begin{abstract}
Background: Staphylococcus epidermidis is the leading coagulase negative staphylococci (CoNS) species associated with healthcare associated infections. In order to de-escalate antimicrobial therapy, isolates of S. epidermidis lacking the blaz gene should be eligible for targeted antimicrobial therapy. However, testing the susceptibility of coagulase negative staphylococci (CoNS) to penicillin $\mathrm{G}$ is no longer recommended by EUCAST, given the low performances for penicillinase detection in CoNS. The objective of this work was to determine a phenotypic method with high performance for detecting penicillinase production in S. epidermidis.

Results: Four techniques for the detection of penicillinase production (disk diffusion, zone edge test, nitrocefin test, Minimal Inhibitory Concentration (MIC) by automated system Vitek2 ${ }^{\circledR}$ ) were evaluated on 182 S. epidermidis isolates, using identification of blaZ gene by PCR as the reference method. The performance of the methods for penicillinase detection was compared by the sensitivity, the specificity, the negative predictive value and the positive predictive value, and with Cohen's kappa statistical test. Among the $182 \mathrm{~S}$. epidermidis included in this study, 55 carried the blaz gene. The nitrocefin test, characterized by a poor sensitivity (91\%), was therefore excluded from $\mathrm{S}$. epidermidis penicillinase detection. The algorithm proposed here for the penicillinase detection in $\mathrm{S}$. epidermidis involved two common antimicrobial susceptibility techniques: disk diffusion method and MIC by Vitek ${ }^{\circledR}$ system. Disk diffusion method, interpreted with a $26 \mathrm{~mm}$ breakpoint for penicillin $\mathrm{G}$, was associated with a high sensitivity (98\%) and specificity (100\%). This method was completed with zone edge test for S. epidermidis with penicillin $\mathrm{G}$ diameter from 26 to $35 \mathrm{~mm}$ (sensitivity of $98 \%$ ). The Vitek $2^{\circledR}$ system is associated with a low sensitivity (93\%) and a high specificity (99\%) This low sensitivity is associated with false negative results, in isolates with 0.12 mg/L Penicillin G MIC values and blaZ positive. Thus for penicillin G MIC of $0.06 \mathrm{mg} / \mathrm{L}$ or $0.12 \mathrm{mg} / \mathrm{L}$, a second step with disc diffusion method is suggested.
\end{abstract}

Conclusions: According to our results, the strategy proposed here allows the interpretation of penicillin $G$ susceptibility in $\mathrm{S}$. epidermidis isolates, with an efficient detection of penicillin $\mathrm{G}$ resistance.

Keywords: Penicillinase detection, Staphylococcus epidermidis, Disk diffusion method, Zone edge test, Nitrocefin test, MIC

\footnotetext{
* Correspondence: helene.pailhories@chu-angers.fr

'Laboratoire de bactériologie, CHU Angers, 4 rue Larrey, 49933 Angers,

France

${ }^{3}$ Laboratoire HIFIH, UPRES EA3859, SFR 4208, Université d'Angers, Angers,

France

Full list of author information is available at the end of the article
}

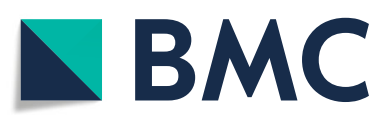

(c) The Author(s). 2020 Open Access This article is licensed under a Creative Commons Attribution 4.0 International License, which permits use, sharing, adaptation, distribution and reproduction in any medium or format, as long as you give appropriate credit to the original author(s) and the source, provide a link to the Creative Commons licence, and indicate if changes were made. The images or other third party material in this article are included in the article's Creative Commons licence, unless indicated otherwise in a credit line to the material. If material is not included in the article's Creative Commons licence and your intended use is not permitted by statutory regulation or exceeds the permitted use, you will need to obtain permission directly from the copyright holder. To view a copy of this licence, visit http://creativecommons.org/licenses/by/4.0/ The Creative Commons Public Domain Dedication waiver (http://creativecommons.org/publicdomain/zero/1.0/) applies to the data made available in this article, unless otherwise stated in a credit line to the data. 


\section{Background}

Staphylococcus epidermidis is the first coagulasenegative Staphylococcus (CoNS) documented in the early twentieth century, with a taxonomic description in 1908 as Albococcus albus [1]. This one is also one of the most frequent CoNS isolated in humans [1]. Indeed, among skin CoNS, S. epidermidis is the most common species colonizing the body surface [2-4]. However, this bacterium is also responsible for healthcare-associated infections (HAI) [5-7]. Indeed, S. epidermidis has been reported as the leading bacterial species associated with device-associated HAI, such as catheters or prosthetic cardiac valves $[1,8,9]$. Native valve endocarditis, infections in neonates and bacteremia in neutropenic patients have also been described [1].

The first resistance of $S$. epidermidis to penicillin has been firstly detected, in four fatal cases of subacute bacterial endocarditis in 1949 [10]. Today, more than $90 \%$ of $S$. epidermidis are considered as resistant to penicillin [11, 12]. This resistance affects penicillins V, G and A, ureidopenicillins and carboxypenicillins. The susceptibility of S. epidermidis to these molecules is restored by the addition of beta-lactamase inhibitors such as clavulanic acid. In addition, many other classes of the beta-lactam family remain sensitive (penicillin $\mathrm{M}$, cephalosporins, carbapenems). A major mechanism of penicillin resistance is penicillinase production, an enzyme that hydrolyzes the beta-lactam ring of penicillin $[13,14]$. This plasmid-mediated beta-lactamase is encoded by the bla $\mathrm{Z}$ gene [15]. The blaZ gene is associated with a repressor gene blaI, and a signal transducer-sensor protein blaR1 [15]. The blaZ gene expression is upregulated following the interaction between blaR1 and beta-lactams, which is responsible for an inactivation of the repressor blaI. The mecA gene can also be mentioned as another gene responsible for penicillin resistance but also for resistance to beta-lactams in general in $S$. epidermidis.

The inducible mechanism of penicillinase production in presence of beta-lactams makes it difficult to identify in CoNS, and thus to conclude on the penicillin resistance. No European recommendations prevail for penicillin susceptibility testing in S. epidermidis, EUCAST stating that no currently available method is reliable for penicillinase production detection in CoNS. All strains must de facto be reported as resistant to benzylpenicillin, ampicillin, amoxicillin, piperacillin and ticarcillin. However, for $S$. aureus, EUCAST recommends the use of disk diffusion method, zone diameter measure and zone edge inspection being more reliable than Minimal Inhibitory Concentration (MIC) determination for the detection of penicillinase [16]. Chromogenic tests are also available for the penicillinase detection, although they are not recommended for this use. Nevertheless, penicillins are of considerable interest because these molecules have few side effects, significant bactericidal properties, a narrow spectrum and low therapeutic costs [14].

The aim of this study was to compare different methods for the diagnosis of penicillinase production and to determine a strategy based on phenotypic methods for asserting the production of penicillinase in S. epidermidis.

\section{Results}

\section{Bacterial isolates}

In this study, 182 non-duplicate methicillin-susceptible S. epidermidis clinical isolates (MSSE) were included. Among them, 127 strains interpreted susceptible to penicillin $G$ and 55 strains resistant to this antibiotic were included [14]. S. epidermidis were mainly isolated from blood cultures (59), urines (44), tissues (14), liquids (20), catheters (20), implantable venous access devices (9), CSF (5) and other superficial samples (11).

\section{blaZ gene detection}

A total of $127 / 182$ isolates (69.8\%) were found blaZ negative while $55 / 182$ isolates (30.2\%) carried the blaZ gene.

\section{Diffusion method - reading inhibition diameters}

Considering the 182 isolates, penicillin $\mathrm{G}$ inhibition zone diameters ranged from 6 to $55 \mathrm{~mm}$. The 55 blaZ positive S. epidermidis isolates displayed diameters between 6 and $26 \mathrm{~mm}$. The 127 blaZ negative isolates formed diameters between 33 and $55 \mathrm{~mm}$ (Fig. 1a).

Using the cut-off value of $26 \mathrm{~mm}$ (EUCAST recommendations for $S$. aureus [16]), the sensitivity of the method was $98 \%$, the specificity of $100 \%$, the negative predictive (NPV) value of $99 \%$ and the positive predictive value (PPV) for the detection of blaZ positive isolates was $100 \%$ (Table 1). One S. epidermidis isolate with a diameter of $26 \mathrm{~mm}$, thus interpreted as susceptible to penicillin $G$ following the guidelines previously mentioned, was in fact blaZ positive. A very strong agreement was observed between penicillin $G$ zone diameters and blaZ PCR $(\kappa=0.987)$.

\section{Diffusion method - nitrocefin disk test}

The 127 blaZ negative S. epidermidis isolates had a negative nitrocefin disk test result (unchanged or yellow colour after $60 \mathrm{~min}$ ). Among the 55 blaZ positive isolates, 50 had a positive result, but 5 had a negative nitrocefin test. These 5 false negative (FN) results were associated with penicillin $G$ inhibition zone diameters ranging from 16 to $20 \mathrm{~mm}$. The nitrocefin disk test had a sensitivity of $91 \%$, a specificity of $100 \%$, a positive predictive value of $100 \%$ and a negative predictive value of 


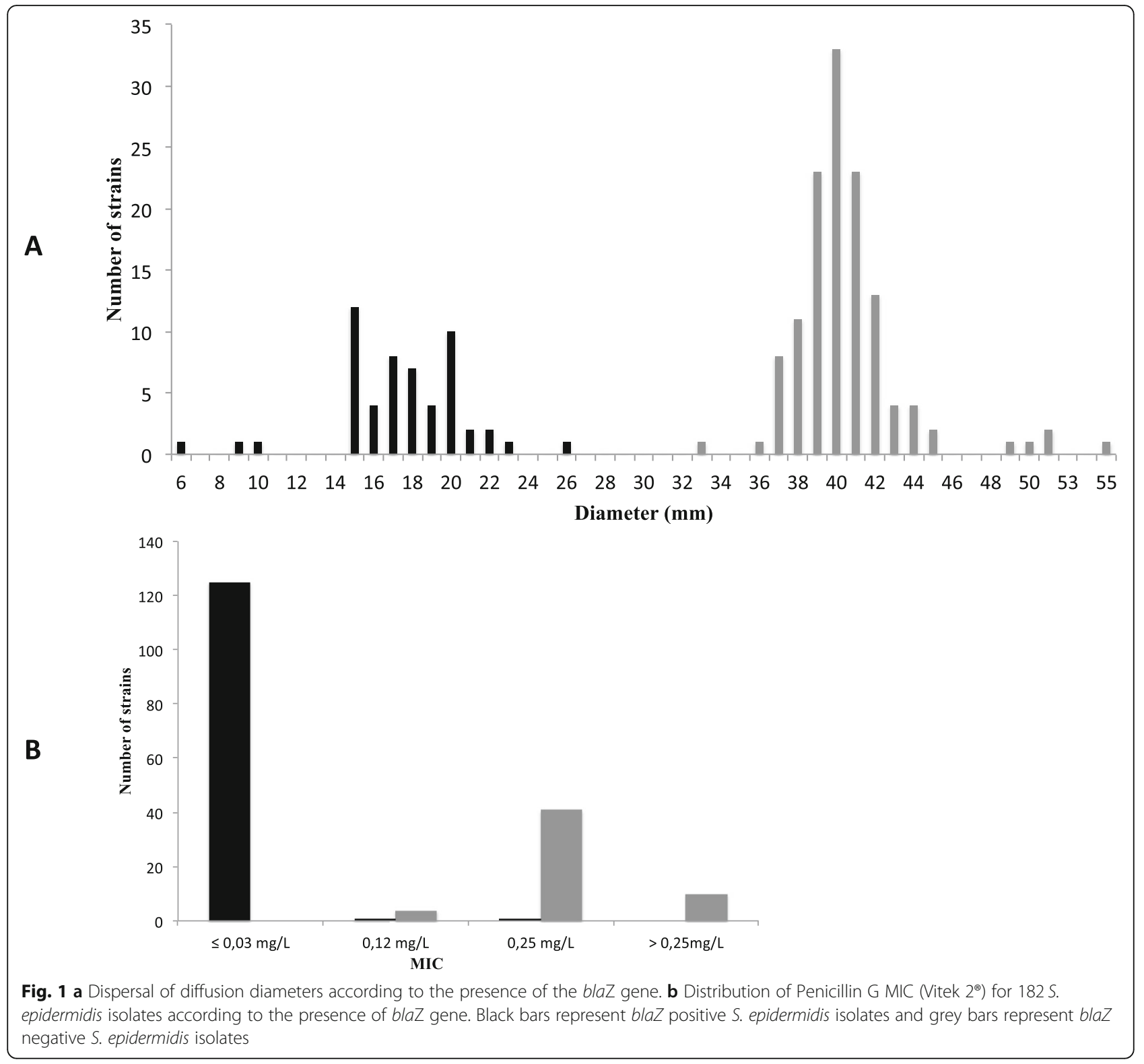

96\% (Table 1). A very strong agreement was observed between nitrocefin disk test and blaZ PCR $(\kappa=0.933)$.

\section{Zone edge test}

To evaluate the performance of this method, we first evaluated the sensitivity and specificity of the zone edge test interpretation of each investigator. Then, the interpretation of all the investigators were combined, selecting the result of the majority of the investigators for each S. epidermidis isolate (at least 3 interpretations within the 5) (Table 1). The 5 investigators selected for zone edge test reading were skilled bacteriologists with a 2 to 25 -year experience.

Sensitivity differed slightly between the five investigators, with a sensitivity greater than or equal to $96 \%$ for all readers (from 96 to 100\%). On the contrary, a wide variation of the specificity was observed, ranging from 66 to $96 \%$ (Table 1). No correlation could be established between the length of the laboratory practice and the performances of zone edge test reading. Combining the results of the five investigators, zone edge test had a sensitivity of $98 \%$, a specificity of $84 \%$, a positive predictive value of $73 \%$ and a negative predictive value of $99 \%$ (Table 1). Indeed, on the 127 blaZ negative $S$. epidermidis isolates, 20 were interpreted with a positive zone edge test, and on the 55 blaZ positive $S$. epidermidis isolates, one was interpreted with a negative zone edge test.). A strong agreement was observed between nitrocefin disk test and blaZ PCR ( $\mathrm{\kappa}=0.751)$. 
Table 1 Performances of Penicillin G zone diameter, nitrocefin disk test, Zone edge test and Penicillin G MIC determination (Vitek $\left.2^{\circledast}\right)$ for the detection of penicillinase production in $182 \mathrm{~S}$. epidermidis isolates and comparison of zone edge test results between the 5 investigators

\begin{tabular}{|c|c|c|c|c|c|c|c|c|c|}
\hline Phenotypic method for penicillinase detection & TP $(N)$ & $\mathrm{FP}(\mathrm{N})$ & $\mathrm{TN}(\mathrm{N})$ & $\mathrm{FN}(\mathrm{N})$ & Total (N) & Se (\%) & $\mathrm{Sp}(\%)$ & PPV (\%) & NPV (\%) \\
\hline Diameter & 54 & 0 & 127 & 1 & 182 & 98 & 100 & 100 & 99 \\
\hline Nitrocefin & 50 & 0 & 127 & 5 & 182 & 91 & 100 & 100 & 96 \\
\hline Zone edge test & 54 & 20 & 107 & 1 & 182 & 98 & 84 & 73 & 99 \\
\hline MIC & 51 & 1 & 126 & 4 & 182 & 93 & 99 & 98 & 97 \\
\hline \multicolumn{10}{|l|}{ zone edge test reading/investigators } \\
\hline Investigator 1 & 54 & 21 & 106 & 1 & 182 & 98 & 83 & 72 & 99 \\
\hline Investigator 2 & 54 & 19 & 108 & 1 & 182 & 98 & 85 & 74 & 99 \\
\hline Investigator 3 & 53 & 43 & 84 & 2 & 182 & 96 & 66 & 55 & 98 \\
\hline Investigator 4 & 55 & 28 & 99 & 0 & 182 & 100 & 78 & 66 & 100 \\
\hline Investigator 5 & 54 & 5 & 122 & 1 & 182 & 98 & 96 & 93 & 99 \\
\hline Final result & 54 & 20 & 107 & 1 & 182 & 98 & 84 & 73 & 99 \\
\hline
\end{tabular}

blaZ PCR was used as the reference method

$T P$ true positive, FP false positive, TN true negative, FN false negative, Se Sensitivity, Sp Specificity, PPV Positive Predictive Value, NPV Negative Predictive Value, ( $N$ ) Number of isolates

\section{Penicillin G MIC - Vitek $2^{\circledR}$ method}

A sensitivity of $93 \%$ and a specificity of $99 \%$ were assessed for this method, with a negative predictive value of $97 \%$, and a positive predictive value of $98 \%$ (Table 1 ). Of the 127 isolates lacking the blaZ gene, 125 corresponded to MICs $\leq 0.03 \mathrm{mg} / \mathrm{L}$, one had a MIC of 0.12 $\mathrm{mg} / \mathrm{L}$, and one had a MIC of $0.25 \mathrm{mgL}$, and was thus considered as a false positive. On the 55 bla $\mathrm{Z}$ positive isolates, 4 had a MIC of $0.12 \mathrm{mg} / \mathrm{L}$ and considered as false negative results, 41 had a MIC of $0.25 \mathrm{mg} / \mathrm{L}$, and 10 with a MIC result $>0.25 \mathrm{mg} / \mathrm{L}$ (Fig. 1b). A very strong agreement was observed between nitrocefin disk test and blaZ PCR $(\mathrm{K}=0.934)$.

\section{Discussion}

The aim of this work was to determine a sensitive and specific phenotypic method for the diagnosis of S. epidermidis penicillinase production. Here, blaZ PCR has been used as the reference technique for the detection of penicillinase. The objective of this study was to assess the performance of different phenotypic methods for the diagnosis of penicillinase production.

The disk diffusion method as used in this study yielded $98 \%$ sensitivity and $100 \%$ specificity results for the detection of S. epidermidis penicillinase production. Few studies exist on the performance of penicillinase detection in CoNS. A study of Kaase et al. also assessed the performance of disk diffusion method for the penicillinase detection, but only on Staphylococcus aureus isolates. In this last work, disk diffusion method was interpreted following CLSI recommendations, using a 10 unit penicillin G disk and interpreted with a zone diameter breakpoint of $28 \mathrm{~mm}$ (diameters $\leq 28 \mathrm{~mm}$ interpreted as resistant to penicillin). A sensitivity of $57 \%$ was obtained for the detection of penicillinase with this method on 197 isolates of S. aureus [17]. Also using CLSI recommendations, Ferreira et al. achieved $72 \%$ sensitivity results in 101 isolates of Staphylococcus (including 17\% of S. aureus and $73 \%$ of CoNS isolates) [18]. The sensitivity results obtained in these studies seem therefore much lower than the result obtained here for $S$. epidermidis isolates. According to the EUCAST recommendations, the susceptibility to penicillin $G$ is determined by using a 1 unit penicillin $G$ disk and an inhibition zone diameter of $26 \mathrm{~mm}$. In a recent study [19], Papanicolas et al. analyzed the production of penicillinase by disk diffusion method on 157 strains of S. aureus by comparing the European and American recommendations. For these strains, the sensitivity and specificity according to the EUCAST recommendations were respectively of 100 and 99\%, as compared to 66 and 100\% with CLSI recommendations. Thus, this last study supports the fact that EUCAST recommendations are a better predictor of beta-lactamase production. This should be taken into account for the analysis of performance of this method for penicillinase detection in $S$. epidermidis. By using EUCAST recommendations for $S$. aureus, good performances were obtained for the detection of S. epidermidis penicillinase in our study. The absence of the bla $\mathrm{Z}$ gene in the 127 strains with a diameter greater or equal to 33 $\mathrm{mm}$ supports the specificity of this test, and highlights the relability to conclude to the susceptibility to penicillin G beyond this diameter. However, the number of $S$. epidermidis isolates with a diameter between 26 and 35 $\mathrm{mm}$ in this study seems insufficient to exclude the presence of bla $Z$ in isolates ranging in these values of penicillin $G$ diameter, especially given the fact that a false negative result has been observed for a blaZ positive $S$. 
epidermidis with a $26 \mathrm{~mm}$ penicillin $\mathrm{G}$ diameter. For this last isolate, zone edge test was positive for the 5 investigators (sharp zone edge), with a positive nitrocefin and a MIC value $>0.25 \mathrm{mg} / \mathrm{L}$. Thus, other diagnosis method should be used for the detection of penicillinase production in S. epidermidis isolates ranging from 26 to $35 \mathrm{~mm}$.

A sensitivity of $91 \%$ and a specificity of $100 \%$ were obtained for the nitrocefin test. The performances of this test for the detection of Staphylococcus penicillinase vary widely in the literature. For $S$. aureus isolates, sensitivity results range from 39 to $92 \%[17,19,20]$. Ferreira et al. also analyzed the performances of this method on different species of Staphylococci, and conclude with a sensitivity of $29 \%$, which is much lower than the performance results obtained in this study [18]. However, we confirm here the results of other reports, outlining a lower sensitivity of nitrocefin test comparing with other phenotypic tests, including zone edge test [18-20]. The great variability of these results makes it difficult to consider this technique as reliable for the detection of beta-lactamase production in Staphylococcus. The performance variation according to studies could also be associated with the different reagents used for the realization of this technique (nitrocefin solution or disks), and to the localization of colonies picked for the test (on penicillin $\mathrm{G}$ zone edge as in this study, on cefoxitin zone edge or without any induction). Here, this test, associated with the lowest negative predictive value within all the phenotypic methods tested (96\%), should not be recommended for the detection of penicillinase in S. epidermidis.

The results of the inspection of the zone edge are contrasted. EUCAST recommends the inspection of penicillin G zone edge in $S$. aureus, for isolates with diameters $\geq 26 \mathrm{~mm}$. Here, we applied this method to all the S. epidermidis isolates. The sensitivity is $98 \%$, but the low specificity result (84\%) seems insufficient to use this method. Indeed, the positive predictive value, associated with the ability of this technique to correctly assign isolates as blaZ positive, is only of $73 \%$. The good sensitivity recorded in our study for this test is supported by the literature. Indeed, a sensitivity greater than $90 \%$ was obtained by testing different species of Staphylococci [18], and a sensitivity of $100 \%$ was recorded by testing different $S$. aureus isolates [19]. In a study of Gill et al. carried out on 260 strains of S. epidermidis, the sensitivity determined for this method was $95 \%$. However, in this same work, the appearance of the zone edge was uninterpretable for 17 S. epidermidis (14 penicillinase producers and 3 non-producers) [21]. This study highlights the difficulties encountered in the interpretation of this test on some S. epidermidis isolates. On the other hand, the subjective aspect of the method (associated with human interpretation) can explain the variability of the results and the low specificity obtained for this test. In a study of Hombach et al. performed on S. aureus isolates, a wide variation in the results of the zone edge test have been observed depending on the level of experience of the investigator. Similarly, the results of the zone edge test varied between the different readers in our study If the sensitivity varied slightly (from 96 to 100\%), the specificity could vary by $30 \%$ between two investigators. Discrepancies between the microbiologists in the interpretation of the zone edge test were noted for approximately one third of $S$. epidermidis isolates (58/182), with a total of 111 interpretation errors. For 56 of these 58 discordant strains, the penicillin $\mathrm{G}$ diameter was greater or equal to $33 \mathrm{~mm}$. Thus, an error has been made by at least one of the microbiologists, on $44 \%$ of the 127 bla $\mathrm{Z}$ negative isolates. These errors seemed therefore relatively recurring between the different investigators. It is interesting to note, however, that for the only S. epidermidis isolate with a discordant result by disk diffusion method (diameter $\geq 26 \mathrm{~mm}$ and blaZ positive), the zone edge test interpretation was concordant for all microbiologists. Furthermore, combining the results of all investigators, an error occurred in $11,5 \%$ of the S. epidermidis isolates mainly consisting in false positive interpretations (20 errors on 21). This phenotypic method therefore should appear as an additional criterion for strains with a penicillin $G$ diameter between 26 and $35 \mathrm{~mm}$. However, the difficulty of zone edge interpretation associated with the subjective nature of this test should be duly noted, so as the risk to slightly overestimate the number of $S$. epidermidis penicillinase producer.

The results of the microdilution method, carried out here with the Vitek $2^{\circ}$ system, were associated with a sensitivity of $93 \%$ and a specificity of $98 \%$. In addition, in our study, and as reported in the literature for $S$. aureus isolates, no strain with MIC values $\leq 0.03 \mathrm{mg} / \mathrm{L}$ carried the blaZ gene [17, 20, 22]. In our study, no strain had a $0.06 \mathrm{mg} / \mathrm{L}$ MIC, 4 out of 5 strains with a MIC of 0.12 $\mathrm{mg} / \mathrm{L}$ and 40 strains out of 41 with a MIC of $0.25 \mathrm{mg} / \mathrm{L}$ were blaZ positive, attesting to the low reliability of this method to detect penicillinase production for MICs between 0.06 and $0.12 \mathrm{mg} / \mathrm{L}$. In a study of Richter et al. including 448 S. aureus strains, $96 \%$ of strains with MIC = $0.06 \mathrm{mg} / \mathrm{L}$ and $68 \%$ of the strains with $\mathrm{MIC}=0.12 \mathrm{mg} / \mathrm{L}$ were blaZ negative. The results of the study conducted by Kaase et al. with $197 \mathrm{~S}$. aureus strains were similar, $94 \%$ of strains with a MIC $=0.06 \mathrm{mg} / \mathrm{L}$ and $77 \%$ of strains with a $\mathrm{MIC}=0.12 \mathrm{mg} / \mathrm{L}$, being blaZ negative. Thus, on the results recorded with strains of $S$. aureus do not seem comparable to those obtained in the present study, with isolates of S. epidermidis. These results prompt us in the context of the study of S. epidermidis to establish a breakpoint of this method to 0.03 $\mathrm{mg} / \mathrm{L}$ to attest the absence of beta-lactamase, and continue testing strains with MIC from 0.06 to $0.12 \mathrm{mg} / \mathrm{L}$ 
with another technique to ensure the absence of production of penicillinase. For laboratories that only have an antimicrobial susceptibility testing system by the microdilution method, these results are important for establishing a decision algorithm for the detection of penicillinase production.

One limitation that could be pointed in this study is using blaZ PCR as the reference method. Indeed, the transcription level of blaZ has not been investigated. Indeed a correlation could maybe have been made between the level of transcription of the blaZ gene and the false positive results obtained with some phenotypic methods. Such investigations should be interesting to perform in further studies.

In summary two methods seem particularly interesting to determine the susceptibility of $S$. epidermidis to penicillin G, the disk diffusion method and MIC Vitek $2^{\circ}$ method. Both methods are available in most bacteriology laboratories, and seem here to have good performance for the detection of penicillinase production in S. epidermidis, but using revised breakpoints. The nitrocefin test is associated with a poor sensitivity. Concurrently, the inspection of the zone edge could play a role in complement of the diameter, especially for isolates with a 26 to $35 \mathrm{~mm}$ diameter, taking into account the overestimation of penicillinase producers with this technique which could avoid some false negative results. This approach is summarized in Fig. 2. For laboratories performing antibiograms by microdilution, the diagnosis approach is also suggested in Fig. 2.

\section{Conclusions}

We provide here an original diagnosis strategy, based on phenotypic methods, for the determination of S. epidermidis susceptibility to Penicillin G. The diagnosis approach proposed here is valid for MSSE isolates, so only after the inspection of the methicillin susceptibility of the $S$. epidermidis isolates. Indeed, in order to deescalate antimicrobial therapy, MSSE isolates lacking the blaZ gene should be eligible for targeted antimicrobial therapy. The diagnosis approach suggested here should allow the interpretation of Penicillin G susceptibility in S. epidermidis isolates.

\section{Methods}

\section{Bacterial isolates}

In this study, 182 non-duplicate methicillin-susceptible S. epidermidis clinical isolates (MSSE), isolated in the laboratory of bacteriology of a French teaching hospital between January 2015 and August 2018, were included. S. epidermidis strains interpreted susceptible to penicillin G - following 2015 to 2017 CA-SFM recommendations (Committee for antibiotic susceptibility testing of the French Society of Microbiology) - were selected [14]. The penicillin G susceptibility of S. epidermidis isolates was evaluated by the determination of the MIC of penicillin G by Vitek 2 method (bioMérieux, Marcy l'Etoile, France) and on the measurement of inhibition zone diameter of penicillin $G$ by disk diffusion method. In addition, S. epidermidis strains resistant to penicillin G, excluding methicillin-resistant $S$. epidermidis, were also included.

\section{Bacterial cultures}

The S. epidermidis isolates were subcultured from Stock Culture storage medium (Bio- $\mathrm{Rad}^{\circ}$, Hercules, USA) on Columbia sheep blood agar (Oxoid ${ }^{\circ}$, Dardilly, France) and incubated $24 \mathrm{~h}$ at $35+/-2{ }^{\circ} \mathrm{C}$. Identification of bacterial colonies was verified by Matrix Assisted Laser Desorption Ionization - Time of Flight mass spectrometry (MALDI-TOF MS) (bioMérieux ${ }^{\circ}$, Marcy l'Etoile, France).

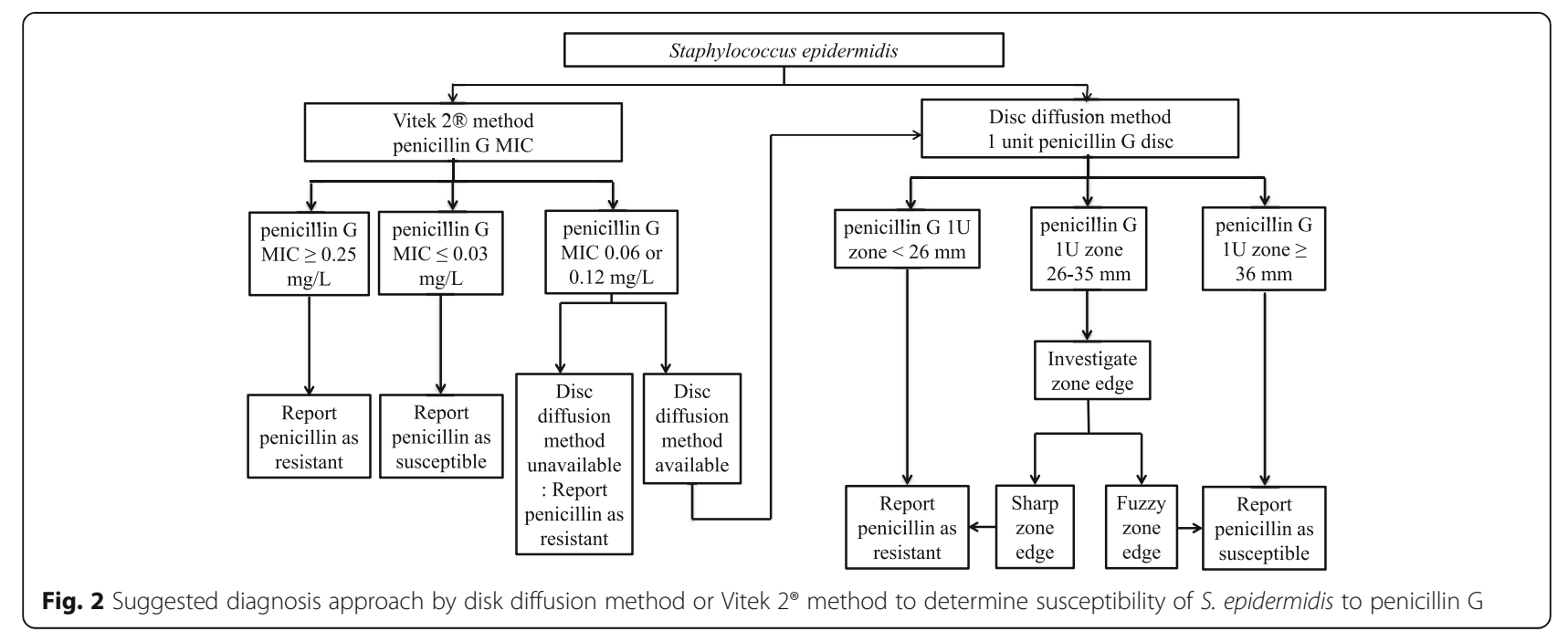




\section{blaZ gene detection}

The DNA was extracted from bacterial colonies using a lysostaphin-based extraction protocol. Genetic detection of blaZ genes was performed by using an inhouse real-time PCR, amplifying a $165 \mathrm{bp}$ fragment of the blaZ gene (nucleotide position 665-830). The total DNA extracted from $S$. epidermidis colonies was amplified by using blaZ -for primer (5'-TGCTGA TAAAAGTGGTCAAGCA-3 '), blaZ-rev primer (5'ACACTCTTGGCGGTTTCACT-3'), blaZ-dye probe (5'-FAM-TCCTAAGGGCCAATCTGAACCTATTGTBHQ1-3') (Eurofins ${ }^{\circ}$ ), Brilliant III ultrafact QPCR Low ROX Master Mix (Agilent ${ }^{\circ}$ ) and water for PCR (Invitrogen ${ }^{\circ}$ ) for a final volume of $20 \mu \mathrm{L}$ for each reaction mix. The PCR protocol was performed on MX 3000 (Agilent ${ }^{\circ}$ ) and included an initial denaturation at $95^{\circ} \mathrm{C}$ for $3 \mathrm{~min}$ followed by 45 denaturation cycles $\left(95^{\circ} \mathrm{C}\right.$ for $\left.15 \mathrm{~s}\right)$ and hybridization / elongation cycles $\left(55^{\circ} \mathrm{C}\right.$ for $\left.25 \mathrm{~s}\right)$. Some blaZ positive and negative $S$. epidermidis strains were used as controls for each PCR run (clinical S. epidermidis isolated in the laboratory previously proved as blaZ negative and positive, and respectively producer and non-producer of penicillinase).

\section{Diffusion method - reading inhibition diameters}

The disk diffusion method was performed on MuellerHinton agar plates $\left(\mathrm{Oxoid}^{\circ}\right.$, Dardilly, France) with a 0.5 Mac Farland bacterial suspension, and by using a 1 unit disk of penicillin G ( $\mathrm{Oxoid}^{\circ}$, Dardilly, France) according to EUCAST recommendations [16]. The plates were incubated $20+/-4 \mathrm{~h}$ at a temperature of $35+/-2{ }^{\circ} \mathrm{C}$. To determine the susceptibility to penicillin, a breakpoint value of $26 \mathrm{~mm}$, following EUCAST recommendations for $S$. aureus species, was used [16].

\section{Diffusion method - nitrocefin disk test}

Nitocefin disk test has been used for penicillinase detection since a long time in bacteriology laboratories on several bacterial species, including Staphylococcus. If this chromogenic based method is no longer recommended by EUCAST for Staphylococci, including S. aureus, the aim here was to state the performance of this method on S. epidermidis isolates. The nitrocefin disk test (Remel ${ }^{\circ}$, San Diego, USA) was used according to the recommendations of the manufacturer. Briefly, colonies picked from the penicillin $G$ inhibition zone edge were applied on a moistened nitrocefin disk. The test was interpreted, according to the indications of the supplier, $60 \mathrm{~min}$ after the beginning of the test and checked for a colour change. A pink/red test has been rated as a positive result, and a yellow or colorless test has been rated as negative.

\section{Zone edge test}

The appearance of the zone edge of penicillin $G$ inhibition zone was also determined. Zone edge test is recommended by EUCAST for penicillinase detection in $S$. aureus isolates with a Penicillin G diameter greater than $26 \mathrm{~mm}$. The aim here was to state the performance of this method in S. epidermidis isolates. Five experienced bacteriologists have inspected the appearance of the zone edges and classified as following: sharp if well defined (therefore resistant to penicillin G) and fuzzy if not clearly delimited (therefore susceptible to penicillin G). Each reading was performed independently without consultation between the investigators. For each S. epidermidis isolate, the results of the five readers have been compiled, and the most frequent interpretation (given by at least 3 investigators) has been chosen as the final result. Thus, the final result for each isolate (fuzzy or sharp) was the result given by the majority of the readers.

\section{Penicillin G MIC - Vitek $2^{\circledR}$ method}

The MICs of penicillin G were initially tested by the microdilution method, with the Vitek2 ${ }^{\circ}$ AST-P631 system (Vitek2 ${ }^{\circ}$, bioMérieux, Marcy l'Etoile, France), testing penicillin $G$ concentrations ranging from $\leq 0.03 \mathrm{mg} / \mathrm{L}$ to $>0.25 \mathrm{mg} / \mathrm{L} \quad(\leq 0.03 \mathrm{mg} / \mathrm{L}, 0.06 \mathrm{mg} / \mathrm{L}$, $0.12 \mathrm{mg} / \mathrm{L}, \quad 0.25 \mathrm{mg} / \mathrm{L}$ and $>0.25 \mathrm{mg} / \mathrm{L}$ ). When a discrepancy was observed between the MIC value of penicillin G and the blaZ PCR result, the MIC was determined a second time by the same technique to confirm this result.

\section{Results interpretation and analysis}

The blaZ gene PCR was considered as the gold standard to attest the presence of a penicillinase. Each method tested in this study was compared with this reference method. Each discrepant result with the reference method (blaZ PCR) has been repeated once for confirmation. The performance of the different methods for penicillinase diagnosis was compared by the sensitivity, the specificity, the negative predictive value and the positive predictive value and a diagnosis approach was suggested considering the performance of the different methods. Furthermore, staistical analyses were performed using Cohen's kappa test (SPSS v15.0). Indeed, a kappa coefficient between 0.81 and 1 indicated a very strong agreement between the results of the test and the blaZ PCR reference test. A kappa coefficient between 0.61 and 0.8 indicated a strong agreement between the two results. Conversely, a kappa coefficient of $<0.61$ meant that the two results were not sufficiently in agreement. 


\section{Abbreviations}

CLSI: Clinical and Laboratory Standards Institute; CoNS: Coagulase negative staphylococci; EUCAST: European Committee on Antimicrobial Susceptibility Testing; FN: False negative; FP: False positive; HAl: Healthcare-associated infections; MIC: Minimal inhibitory concentration; MSSE: Methicillinsusceptible Staphylococcus epidermidis; NPV : Negative predictive value; PCR : Polymerase chain reaction; PPV : Positive predictive value

\section{Acknowledgements}

Not applicable.

\section{Authors' contributions}

BA performed the experiments, analyzed the data and wrote the manuscript. $\mathrm{CL}$ and $\mathrm{RC}$ participated in the experiments and in the interpretation of the data. MK participated in the revision of the manuscript. ME and HP participated in the writing and the revisions of the manuscript and the supervision of the project. All authors have read and approved the manuscript.

\section{Funding}

None.

\section{Availability of data and materials}

The datasets used and/or analysed during the current study are available from the corresponding author on reasonable request.

\section{Ethics approval and consent to participate}

Not applicable.

\section{Consent for publication}

Not applicable.

\section{Competing interests}

The authors declare that they have no competing interests.

\section{Author details}

${ }^{1}$ Laboratoire de bactériologie, CHU Angers, 4 rue Larrey, 49933 Angers, France. ${ }^{2}$ CRCINA, Inserm, Université de Nantes, Université d'Angers, 44200 Angers, Nantes, France. ' ${ }^{3}$ Laboratoire HIFIH, UPRES EA3859, SFR 4208, Université d'Angers, Angers, France.

Received: 16 April 2020 Accepted: 29 July 2020

Published online: 05 August 2020

\section{References}

1. Becker K, Heilmann C, Peters G. Coagulase-negative staphylococci. Clin Microbiol Rev. 2014;27:870-926. https://doi.org/10.1128/CMR.00109-13.

2. Bouchiat C, Dupieux C, Garnier F, Denis F, Vandenesch F. Familles des Staphylococcaceae et Micrococcaceae. In: Bactériologie médicale; 2016. p. 261-71.

3. Grice EA, Kong HH, Conlan S, Deming CB, Davis J, Young AC, et al. Topographical and temporal diversity of the human skin microbiome. Science. 2009;29(324):1190-2. https://doi.org/10.1126/science.1171700.

4. Grice EA, Segre JA. The skin microbiome. Nat Rev Microbiol. 2011;9:244-53. https://doi.org/10.1038/nrmicro2537.

5. Otto M. Staphylococcus epidermidis - the "accidental" pathogen. Nat Rev Microbiol. 2009;7:555-67. https://doi.org/10.1038/nrmicro2182.

6. Argemi X, Hansmann Y, Prola K, Prévost G. Coagulase-negative staphylococci Pathogenomics. Int J Mol Sci. 2019;20(5):1215.

7. Shelburne S, Dib R, Endres B, Reitzel R, Li X, Kalia A, et al. Whole-genome sequencing of Staphylococcus epidermidis bloodstream isolates from a prospective clinical trial reveals that complicated bacteraemia is caused by a limited number of closely related sequence types. Clin Microbiol Infect. 2020;26(5):646.e1-8.

8. Uçkay I, Pittet D, Vaudaux P, Sax H, Lew D, Waldvogel F. Foreign body infections due to Staphylococcus epidermidis. Ann Med. 2009:41:109-19. https://doi.org/10.1080/07853890802337045

9. Heilmann C, Ziebuhr W, Becker K. Are coagulase-negative staphylococci virulent? Clin Microbiol Infect. 2019;25(9):1071-80.

10. Griffith GC, Levinson DC. Subacute bacterial endocarditis-a report on 57 patients treated with massive doses of penicillin. Calif Med. 1949;71:403-8.
11. Wang J, Zhang H, Yan J, Zhang T. Literature review on the distribution characteristics and antimicrobial resistance of bacterial pathogens in neonatal sepsis. J Matern Fetal Neonatal Med. 2020;1:1-10.

12. Guo Y, Ding Y, Liu L, Shen $X$, Hao Z, Duan J, et al. Antimicrobial susceptibility, virulence determinants profiles and molecular characteristics of Staphylococcus epidermidis isolates in Wenzhou, eastern China. BMC Microbiol. 2019;19:1.

13. Kirby WMM. Extraction of a highly potent penicillin inactivator from penicillin resistant staphylococci. Science. 1944;99:452-3. https://doi.org/10. 1126/science.99.2579.452.

14. Drugeon H. Bêta-lactamines et Staphylocoques. In: Antibiogramme; 2006. p. $117-24$

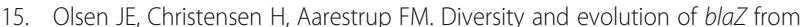
Staphylococcus aureus and coagulase-negative staphylococci. J Antimicrob Chemother. 2006:57:450-60. https://doi.org/10.1093/jac/dki492.

16. EUCAST. Clinical breakpoints and dosing of antibiotics; 2020. http://www. eucast.org/clinical_breakpoints/.

17. Kaase M, Lenga S, Friedrich S, Szabados F, Sakinc T, Kleine B, et al. Comparison of phenotypic methods for penicillinase detection in Staphylococcus aureus. Clin. Microbiol. Infect. Off. Publ. Eur. Soc. Clin. Microbiol. Infect. Dis. 2008;14: 614-6. https://doi.org/10.1111/j.1469-0691.2008.01997.x.

18. Ferreira AM, Martins KB, da Silva VR, Mondelli AL, Cunha MD. Correlation of phenotypic tests with the presence of the blaZ gene for detection of betalactamase. Braz J Microbiol. 2017;48:159-66. https://doi.org/10.1016/j.bjm. 2016.10.011

19. Papanicolas LE, Bell JM, Bastian I. Performance of phenotypic tests for detection of penicillinase in Staphylococcus aureus isolates from Australia. J Clin Microbiol. 2014;52:1136-8. https://doi.org/10.1128/JCM.03068-13.

20. Hombach M, Weissert C, Senn MM, Zbinden R. Comparison of phenotypic methods for the detection of penicillinase in Staphylococcus aureus and proposal of a practical diagnostic approach. J Antimicrob Chemother. 2017; 72:1089-93. https://doi.org/10.1093/jac/dkw521.

21. Gill VJ, Manning CB, Ingalls CM. Correlation of penicillin minimum inhibitory concentrations and penicillin zone edge appearance with staphylococcal Beta-lactamase production. J Clin Microbiol. 1981:14(4):437.

22. Richter SS, Doern GV, Heilmann KP, Miner S, Tendolkar S, Riahi F, et al. Detection and prevalence of penicillin-susceptible Staphylococcus aureus in the United States in 2013. J Clin Microbiol. 2016;54:812-4. https://doi.org/10 1128/JCM.03109-15.

\section{Publisher's Note}

Springer Nature remains neutral with regard to jurisdictional claims in published maps and institutional affiliations.

Ready to submit your research? Choose BMC and benefit from:

- fast, convenient online submission

- thorough peer review by experienced researchers in your field

- rapid publication on acceptance

- support for research data, including large and complex data types

- gold Open Access which fosters wider collaboration and increased citations

- maximum visibility for your research: over $100 \mathrm{M}$ website views per year

At $\mathrm{BMC}$, research is always in progress.

Learn more biomedcentral.com/submissions 